\title{
Swept-Source Optical Coherence Tomographic Findings of Choroidal Osteoma
}

\author{
Yuki Hayashi $^{a}$ Yoshinori Mitamura $^{a}$ Mariko Egawa $^{a} \quad K^{2}$ taro Semba ${ }^{a}$ \\ Toshihiko Nagasawa ${ }^{a, b}$ \\ ${ }^{a}$ Department of Ophthalmology, Institute of Health Biosciences, The University of \\ Tokushima Graduate School, Tokushima, and ${ }^{b}$ Department of Ophthalmology, Saneikai \\ Tsukazaki Hospital, Himeji, Japan
}

\section{Key Words}

Choroidal osteoma - Fundus autofluorescence $\cdot$ Swept-source optical coherence tomography

\begin{abstract}
Purpose: To report the morphologic features of a choroidal osteoma using swept-source optical coherence tomography (SS-OCT) and fundus autofluorescence (FAF). Methods: Two eyes of two cases with a choroidal osteoma were studied using SS-OCT and FAF. Results: The location of the tumor was circumpapillary without macular involvement in case 1 and juxtapapillary with macular involvement in case 2. Both cases had a mixture of calcified and decalcified areas, and a concomitant choroidal neovascularization was found in case 2 . The FAF images showed decreased autofluorescence in the central decalcified regions and relatively preserved fluorescence in marginal calcified regions in both cases. SS-OCT revealed a normal inner retina and an abnormal outer retina in both cases, and subretinal fluid in case 2. The calcified regions appeared sponge-like and were multilayered in case 2. A lamellar reflective pattern was observed in the decalcified regions in case 1 , and hyperreflective mound-like areas were observed in both cases. SS-OCT demonstrated hyperreflective areas above Bruch's membrane accompanied by disruption of Bruch's membrane in case 1 . The chorioscleral border was visible in both cases. Conclusions: The FAF pattern in the calcified and decalcified areas of the choroidal osteoma may correspond to the different stage of tumor evolution. The SS-OCT findings indicate that choroidal osteomas can have characteristic reflective patterns and alterations of the overlying retina.

(c) 2014 S. Karger AG, Basel
\end{abstract}


Hayashi et al:: Swept-Source Optical Coherence Tomographic Findings of Choroidal Osteoma

\section{Introduction}

A choroidal osteoma is a rare ossified tumor of the choroid, and its clinical features were first reported by Gass et al. [1]. The typical features of choroidal osteoma are well-defined borders, slightly elevated and yellowish-white to orange lesions in the peripapillary or macular areas of young healthy women [1]. The color of a choroidal osteoma depends on the degree of thinning and depigmentation of the overlying retinal pigment epithelium (RPE) [2]. Due to the calcification, choroidal osteomas have high reflectivity and acoustic shadowing in ultrasonograms, and they appear as hyperdense plaques at the level of the choroid on computed tomography (CT) images. Although benign, some patients have severe visual loss caused by atrophy of the overlying retina, choroidal neovascularization (CNV), and accumulation of subretinal fluid (SRF) [3]. To treat a choroidal osteoma effectively, it is necessary to identify it and understand its pathogenesis.

One method that has been used to identify a choroidal lesion as an osteoma is optical coherence tomography (OCT). There have been several time-domain or spectral-domain OCT studies of choroidal osteomas [2, 4-9]. However, the obtained images failed to show the morphology in detail because of the depth limitations of these instruments. The recent introduction of swept-source OCT (SS-OCT) has enabled clinicians to obtain a better view of the choroid because of its low sensitivity roll-off with increasing depth compared to spectraldomain OCT $[10,11]$.

We present two cases of choroidal osteoma evaluated by SS-OCT and describe the characteristic findings within the tumor and the overlying outer retina.

\section{Methods}

The diagnosis of choroidal osteoma was based on the presence of a yellowish-white to orangish-red choroidal mass with well-defined margins and bone density reflection in the CT images. Both of our cases were examined with a SS-OCT instrument (DRI OCT-1; Topcon, Tokyo, Japan) which has a wavelength tunable laser centered at 1,050 $\mathrm{nm}$ with a tuning range of approximately $100 \mathrm{~nm}$ as the light source [11]. The tissue imaging depth was 2.6 $\mathrm{mm}$.

Fundus autofluorescence (FAF) was performed with the Topcon TRC-50DX Retinal Camera (Topcon) with an excitation band pass filter of 535-585 $\mathrm{nm}$ and a barrier band pass filter of $615-715 \mathrm{~nm}[12,13]$.

\section{Case Presentations}

Case 1

A 60-year-old man presented with monocular diplopia in his right eye. His family medical history was unremarkable. His best-corrected visual acuity (BCVA) was 1.2 in both eyes, and the intraocular pressure was $11 \mathrm{~mm} \mathrm{Hg}$ in the right eye and $17 \mathrm{~mm} \mathrm{Hg}$ in the left eye. The anterior segment and ocular media were normal in both eyes. The fundus was normal in the left eye.

Examination of the fundus of the right eye demonstrated a 4-disc diameter orangishyellow to yellowish-white circumpapillary lesion (fig. 1a). FAF showed decreased autofluorescence in the decalcified regions of the mass with relatively well preserved autofluorescence in the marginal calcified regions (fig. 1b). Fluorescein angiography (FA) showed that 
Hayashi et al:: Swept-Source Optical Coherence Tomographic Findings of Choroidal Osteoma

the lesion was hyperfluorescent in the early phase with increased staining in the late phase. Indocyanine green angiography showed hypofluorescence through all phases probably because of a loss of the choriocapillaris. B-scan ultrasonography showed a highly echogenic lesion, and CT showed bone density plaques in the right eye. These findings are consistent with a choroidal osteoma.

In the decalcified regions, SS-OCT showed a lamellar reflective pattern (fig. 2a) and hyperreflective mound-like areas (fig. $2 \mathrm{~b}$ ). The hyperreflective regions were observed in the peripapillary choroid. In some regions, the hyperreflective area was located above the Bruch membrane (fig. 2b), and the overlying inner retina was normal but the outer retina was altered. In the outer retina, disruptions of the external limiting membrane (ELM) and the photoreceptor inner/outer segment (IS/OS) junction line were observed (fig. 2b). Disruptions of Bruch's membrane and RPE were also detected (fig. 2c). The vascular layer of the choroid was compressed, and blood vessels were detected in the osteoma (fig. 2a, b). Hyporeflective vertical structures that may correspond to a posterior ciliary vessel penetrating the sclera (fig. 2c) were also seen.

Case 2

A 23-year-old woman presented with myodesopsia in her right eye. Her family medical history was unremarkable. The BCVA was 1.2 in both eyes, and the intraocular pressure was $13 \mathrm{~mm} \mathrm{Hg}$ in the right eye and $19 \mathrm{~mm} \mathrm{Hg}$ in the left eye. The anterior segment and ocular media were normal in both eyes. The fundus was normal in the left eye. Fundus examination of the right eye showed a 7-disc diameter orangish-yellow to white lesion in the posterior pole. B-scan ultrasonography showed a highly reflective lesion in the posterior pole, while CT showed bone density plaques in the right eye. On the basis of these findings, the patient was diagnosed with choroidal osteoma.

One month later, SRF developed and the decimal BCVA decreased to 0.5. She received an intravitreal injection of $1.25 \mathrm{mg} / 0.05 \mathrm{ml}$ of bevacizumab (Avastin ${ }^{\circledR}$; Genentech, South San Francisco, Calif., USA), but the SRF was not reduced. Although we recommended photodynamic therapy as an additional treatment, the patient refused to receive photodynamic therapy. Therefore, two monthly intravitreal injections of bevacizumab were added.

Five months after the first visit, the BCVA was 0.6. The tumor size did not change, but CNV developed (fig. 1c). FAF showed reduced autofluorescence in the decalcified tumor regions and preserved or slightly hyperautofluorescence in the calcified regions (fig. 1d). Fine hyperfluorescence spots were seen in the areas with serous retinal detachments. FA showed leakage corresponding to a CNV (fig. 1e), and indocyanine green angiography showed a thick vascular network in the tumor.

In the regions of calcifications, SS-OCT showed a typical sponge-like pattern composed of dense hyperreflective dots spread through a hyporeflective matrix (fig. 3a). In the decalcified regions, SS-OCT showed hyperreflective mound-like areas (fig. 3a). A multilayer structure, probably due to the presence of different degrees of calcification within the tumor, was found (fig. 3c).

A CNV with SRF was present just beneath the fovea (fig. 3b), and blood vessels were found in the tumor (fig. 3b). The chorioscleral border was detectable despite the presence of SRF (fig. 3a-c), a thick choroid (fig. 3a, c), and its peripapillary location (fig. 3c). The inner retina was normal but disruptions of the ELM and IS/OS junction line were found in the outer retina. Disruptions of the RPE and compression of the choroidal vascular layer were also observed. 
Hayashi et al:: Swept-Source Optical Coherence Tomographic Findings of Choroidal Osteoma

\section{Discussion}

An earlier histopathologic study of choroidal osteomas showed that they were located between the choriocapillaris and the outer choroidal tissue $[1,14]$. The tumor can grow and replace the full thickness of the choroid with decalcification occurring in up to $46 \%$ of the patients in 10 years [15]. The calcified parts appear thicker and more orange, whereas the decalcified parts appear as thin, atrophic, yellowish-gray regions within the osteoma [6]. A decalcification of a choroidal osteoma leads to gradual atrophy of the tumor and poor visual acuity [15]. Therefore, it is important to determine the degree of calcification of the tumor.

Recently, Navajas et al. [2] performed multi-imaging analyses of choroidal osteomas and described a latticework reflective pattern in the spectral-domain OCT images. The images of osteomas were characterized as multiple hyperreflective dots surrounding hyporeflective cavernous spaces in the calcified regions. Pellegrini et al. [8] also described a similar spongelike pattern in the calcified regions. In our case 2, SS-OCT showed the presence of many tiny hyperreflective dots spread into a hyporeflective matrix creating a sponge-like appearance in the marginal calcified regions (fig. 3a). This sponge-like pattern is consistent with a previous histopathologic study that revealed spongy bone consisting of dense bony trabeculae surrounding marrow spaces with loose connective tissue and vessels [14].

A second SS-OCT pattern identified in the calcified area showed multiple reflective layers (fig. 3c). Ide et al. [4] also reported tracks of high refractivity areas posterior to the tumor, although the resolution of the structures was insufficient because of the use of timedomain OCT. Pellegrini et al. [8] described a similar pattern using enhanced depth imaging OCT. In a histopathologic examination, the bony trabeculae tended to extend transversely within the tumor $[1,14]$. There is a possibility that these bony trabeculae arranged in the crosswise pattern may be observed as the multiple intralesional layers which we were able to detect within the tumor by SS-OCT.

Navajas et al. [2] described two patterns of reflectivity in the decalcified areas: the first pattern corresponded to relatively hyperreflective areas with a lamellar appearance, and the second pattern to hyperreflective mound-like areas. We also observed these two patterns of reflectivity in the decalcified areas. In lamellar-appearing regions, no reflective signals corresponding to intratumoral vessels could be identified. Navajas et al. [2] suggested that the lamellar reflective pattern may indicate small amounts of residual tumoral tissue after atrophy of the tumor. They also suggested that the hyperreflective mound-like areas may correspond to tumoral regions in which the spongy organization was lost secondary to partial decalcification.

In the FAF images, the fluorescence pattern was relatively well preserved in the marginal calcified areas, while the central decalcified areas had reduced fluorescence (fig. 1b, d). These OCT and FAF findings may be helpful in evaluating the degree of decalcification of the tumor. We suggest that slightly hyperautofluorescence demonstrated within the calcified parts of the lesion may be due to the RPE surrounding the tumor.

An earlier histopathologic study showed that the location of a choroidal osteoma was between the choriocapillaris and outer choroidal tissue [1,14]. Navajas et al. [2] suggested that the hyperreflective material above Bruch's membrane may be due to extensive fibrous RPE metaplasia because it was very unlikely that the choroidal osteoma grew over it unless the Bruch membrane was disrupted by the tumor. However, we suggest that these hyperreflective areas above Bruch's membrane (fig. 2b) may be tumor-like tissue because we demonstrated a clear disruption of Bruch's membrane (fig. 2c). The tumor-like tissue may have disrupted Bruch's membrane and progressed over it. In addition, several 
Hayashi et al:: Swept-Source Optical Coherence Tomographic Findings of Choroidal Osteoma

intratumor vessels were seen in these hyperreflective areas above Bruch's membrane. Our findings suggest that a choroidal osteoma can grow over Bruch's membrane.

To the best of our knowledge, this is the first SS-OCT analysis of eyes affected by choroidal osteoma. The previous OCT images have been limited because of the insufficient resolution of the detailed structures of the tumor. SS-OCT was able to demonstrate a chorioscleral border in the deep choroidal region (fig. 3), and also in the vessels penetrating the sclera in the peripapillary regions (fig. 2c).

In conclusion, SS-OCT can demonstrate the characteristic reflective patterns in the calcified and decalcified regions of a choroidal osteoma. An intratumoral structure of choroidal osteomas including deeper structures of the eye could be clearly observed. FAF demonstrated different fluorescence patterns in the calcified and decalcified choroidal osteoma.

\section{Disclosure Statement}

The authors have no conflicts of interest to disclose.

\section{References}

1 Gass JD, Guerry RK, Jack RL, Harris G: Choroidal osteoma. Arch Ophthalmol 1978;96:428-435.

-2 Navajas EV, Costa RA, Calucci D, Hammoudi DS, Simpson ER, Altomare F: Multimodal fundus imaging in choroidal osteoma. Am J Ophthalmol 2012;153:890-895.

-3 Alyward GW, Chang TS, Pautler SE, Gass JD: A long-term follow up of choroidal osteoma. Arch Ophthalmol 1998;116:1337-1341.

4 Ide T, Ohguro N, Hayashi A, Yamamoto S, Nakagawa Y, Nagae Y, Tano Y: Optical coherence tomography patterns of choroidal osteoma. Am J Ophthalmol 2000;130:131-134.

-5 Fukasawa A, Iijima H: Optical coherence tomography of choroidal osteoma. Am J Ophthalmol 2002;133:419-421.

6 Shields CL, Perez B, Materin MA, Mehta S, Shields JA: Optical coherence tomography of choroidal osteoma in 22 cases: evidence for photoreceptor atrophy over the decalcified portion of the tumor. Ophthalmology 2007;114:e53-e58.

7 Freton A, Finger PT: Spectral domain-optical coherence tomography analysis of choroidal osteoma. Br J Ophthalmol 2012;95:224-228.

8 Pellegrini M, Invernizzi A, Giani A, Staurenghi G: Enhanced depth imaging optical coherence tomography features of choroidal osteoma. Retina 2013, Epub ahead of print.

-9 Erol MK, Coban DT, Ceran BB, Bulut M: Enhanced depth imaging optical coherence tomography and fundus autofluorescence findings in bilateral choroidal osteoma: a case report. Arq Bras Oftalmol 2013;76:189191.

10 Nagasawa T, Mitamura Y, Katome T, Nagasato D, Tabuchi H: Swept-source optical coherence tomographic findings in morning glory syndrome. Retina 2014;34:206-208.

-11 Nagasawa T, Mitamura Y, Katome T, Shinomiya K, Naito T, Nagasato D, Shimizu Y, Tabuchi H, Kiuchi Y: Macular choroidal thickness and volume in healthy pediatric individuals measured by swept-source optical coherence tomography. Invest Ophthalmol Vis Sci 2013;54:7068-7074.

12 Egawa M, Mitamura Y, Hayashi Y, Naito T: Spectral-domain optical coherence tomographic and fundus autofluorescence findings in eyes with primary intraocular lymphoma. Clin Ophthalmol 2014;8:335-341.

13 Egawa M, Mitamura Y, Hayashi Y, Semba K, Naito T: Changes of fundus autofluorescence and spectraldomain optical coherence tomographic findings after treatment of primary intraocular lymphoma. J Ophthalmic Inflamm Infect 2014;4:7.

$\$ 14$ Williams AT, Font RL, Van Dyk HJ, Riekhof FT: Osseous choristoma of the choroid simulating a choroidal melanoma. Association with a positive 32P test. Arch Ophthalmol 1978;96:1874-1877.

-15 Shields CL, Sun H, Demirci H, Shields JA: Factors predictive of tumor growth, tumor decalcification, choroidal neovascularization, and visual outcome in 74 eyes with choroidal osteoma. Arch Ophthalmol 2005;123:1658-1666. 
Hayashi et al:: Swept-Source Optical Coherence Tomographic Findings of Choroidal Osteoma
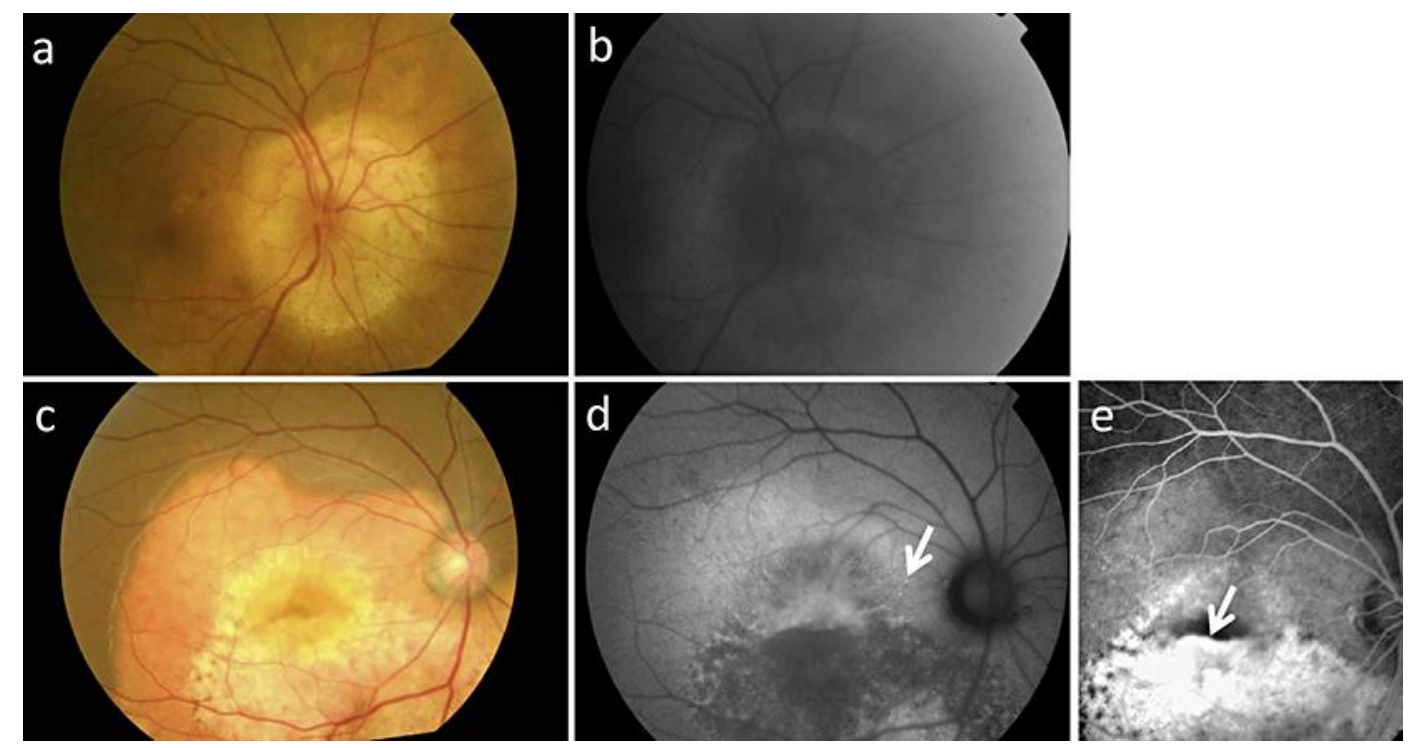

Fig. 1. Color fundus photographs and FAF findings of case $1(\mathbf{a}, \mathbf{b})$ and case $2(\mathbf{c}, \mathbf{d})$, and FA of case 2 (e). Both cases had a choroidal osteoma. a Fundus photograph showing a 4-disc diameter orangish-yellow or yellowish-white circumpapillary lesion in the right eye. Only a minor rim of calcification is observed at the tumor margin. b FAF showing decreased fluorescence in the decalcified regions with relatively well preserved fluorescence in the marginal calcified regions. c Fundus photograph showing a 7-disc diameter orangish-yellow to white lesion in the posterior pole of the right eye. d FAF showing decreased fluorescence in the decalcified regions but preserved or slightly hyperfluorescence in the calcified regions. Fine hyperfluorescent spots (arrow) are evident in the areas with a serous retinal detachment. e FA showing leakage corresponding to CNV (arrow). 


\begin{tabular}{l|l}
\hline \multicolumn{2}{l}{ Case Rep Ophthalmol 2014;5:195-202 } \\
\hline DOI: $10.1159 / 000365184$ & $\begin{array}{l}\text { @ 2014 S. Karger AG, Basel } \\
\text { www.karger.com/cop }\end{array}$ \\
\hline
\end{tabular}

Hayashi et al.: Swept-Source Optical Coherence Tomographic Findings of Choroidal Osteoma
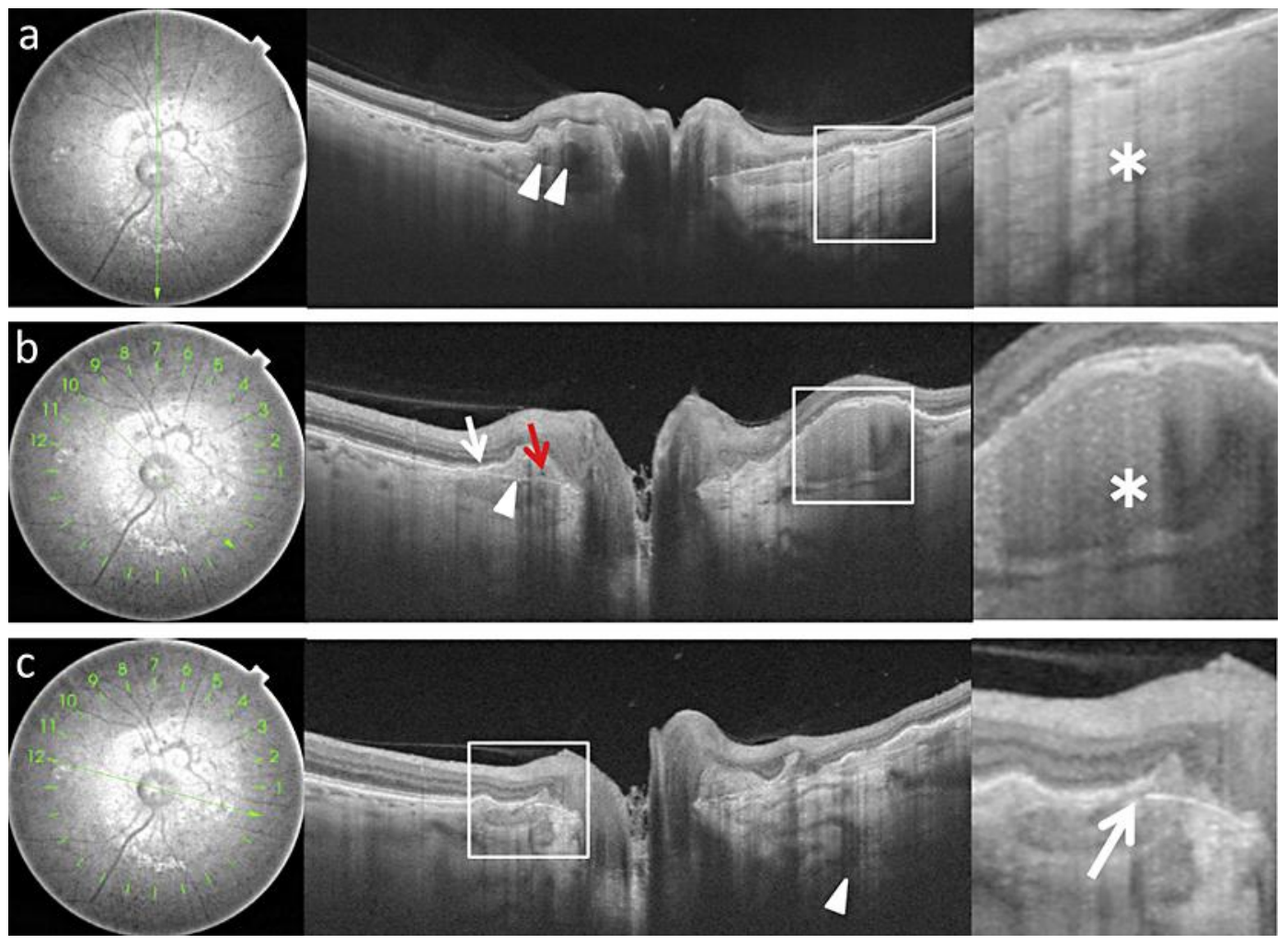

Fig. 2. SS-OCT findings of case 1 . The green arrows in the left column indicate the direction of the OCT scans. The right column shows magnified SS-OCT images in the areas of the square boxes. a A lamellar reflective pattern is observed in a decalcified region (square box and asterisk). Intratumoral vessels are observed (arrowheads). b A hyperreflective area above the Bruch membrane is observed (arrowhead). An intratumoral vessel is observed above the Bruch membrane (red arrow). Disruption of ELM and photoreceptor IS/OS junction can be seen (white arrow). Hyperreflective, mound-like areas are found in the decalcified tumoral regions (square box and asterisk). c Disruption of Bruch's membrane is observed (square box and arrow). Note a hyporeflective vertical structure (arrowhead) that may correspond to a posterior ciliary vessel penetrating the sclera. 


\begin{tabular}{l|l}
\hline \multicolumn{2}{l}{ Case Rep Ophthalmol 2014;5:195-202 } \\
\hline DOI: $10.1159 / 000365184$ & $\begin{array}{l}\text { @ 2014 S. Karger AG, Basel } \\
\text { www.karger.com/cop }\end{array}$ \\
\hline
\end{tabular}

Hayashi et al:: Swept-Source Optical Coherence Tomographic Findings of Choroidal Osteoma
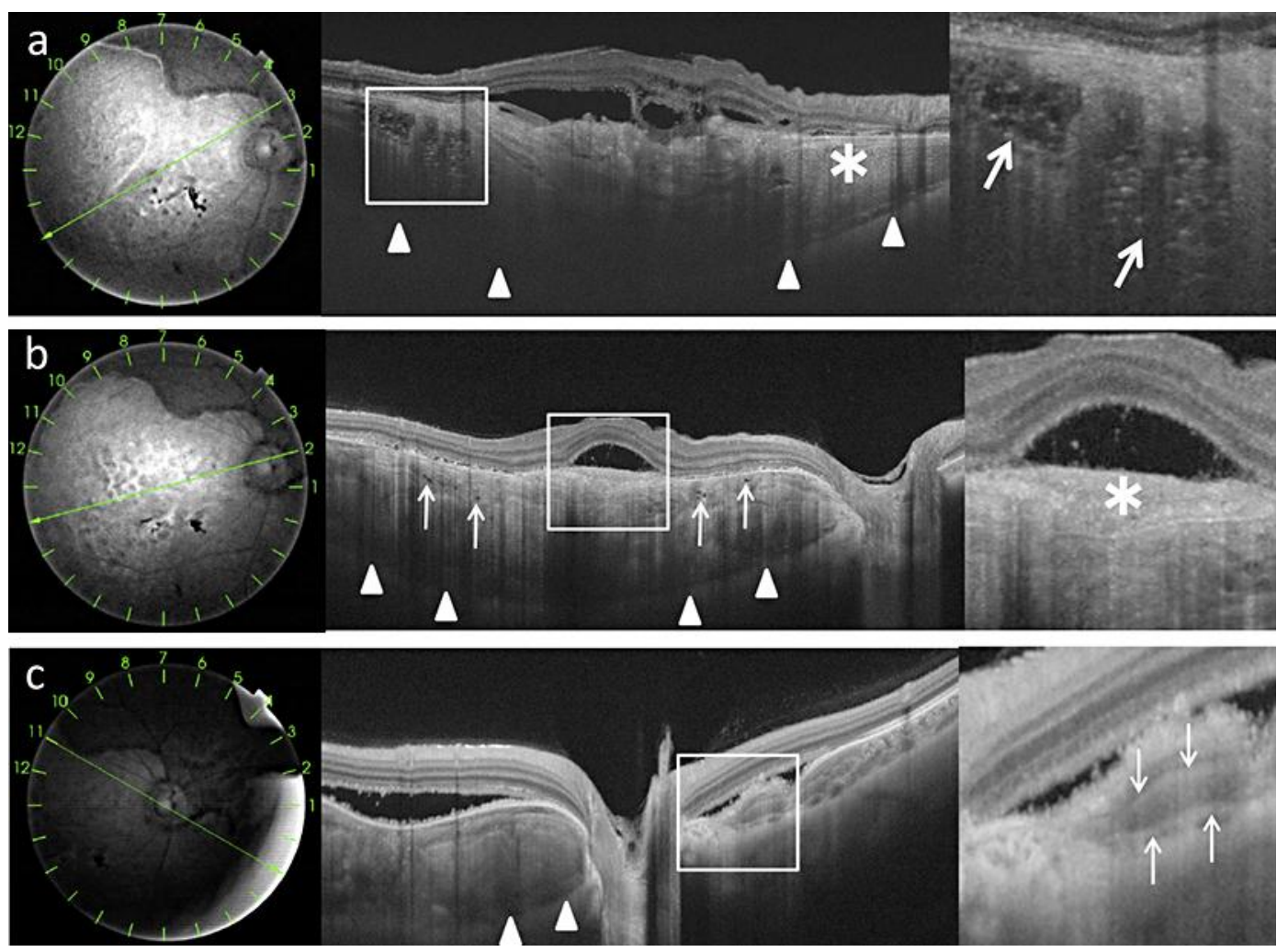

Fig. 3. SS-OCT findings of case 2. The green arrows in the left column indicate the direction of the OCT scans. The right column shows magnified SS-OCT images in the areas of the square boxes. a A sponge-like pattern is seen in the calcified tumoral regions (square box). Hyperreflective, mound-like areas are found in the decalcified tumoral regions (asterisk). Analysis of magnified SS-OCT images reveals a typical sponge-like pattern comprised of dense hyperreflective dots spread into a hyporeflective matrix (arrows). b CNV with SRF is present just beneath the fovea (square box and asterisk). Intratumoral vessels are observed within the tumor (arrows). c Multiple intralesional layers probably caused by the presence of different degrees of calcification within the tumor (square box and arrows). The chorioscleral border (arrowheads) is visible in spite of the presence of SRF (a-c), thick choroid $(\mathbf{a}, \mathbf{b})$, or peripapillary location (c). 